\title{
Microglia modulation with 1070-nm light attenuates $A \beta$ burden and cognitive impairment in Alzheimer's disease mouse model
}

\author{
Lechan Tao', Qi Liu', Fuli Zhang ${ }^{1}$, Yuting Fu' ${ }^{1}$ Xi Zhu' ${ }^{1}$ Xiaofu Weng ${ }^{1}$, Hongbin Han²,3,4 Yong Huang ${ }^{5}$, \\ Yuanzhen Suo $\mathbb{C}^{6,7}$, Liang Chen ${ }^{8,9 凶}$, Xiaoling Gao ${ }^{10 凶}$ and Xunbin Wei ${ }^{1,2,11,12 凶}$
}

\begin{abstract}
Photobiomodulation, by utilizing low-power light in the visible and near-infrared spectra to trigger biological responses in cells and tissues, has been considered as a possible therapeutic strategy for Alzheimer's disease (AD), while its specific mechanisms have remained elusive. Here, we demonstrate that cognitive and memory impairment in an $\mathrm{AD}$ mouse model can be ameliorated by 1070-nm light via reducing cerebral $\beta$-amyloid (A $\beta$ ) burden, the hallmark of $A D$. The glial cells, including microglia and astrocytes, play important roles in $A \beta$ clearance. Our results show that 1070-nm light pulsed at $10 \mathrm{~Hz}$ triggers microglia rather than astrocyte responses in AD mice. The 1070-nm lightinduced microglia responses with alteration in morphology and increased colocalization with $A \beta$ are sufficient to reduce $A \beta$ load in AD mice. Moreover, $1070-\mathrm{nm}$ light pulsed at $10 \mathrm{~Hz}$ can reduce perivascular microglia and promote angiogenesis to further enhance $A \beta$ clearance. Our study confirms the important roles of microglia and cerebral vessels in the use of 1070-nm light for the treatment of $A D$ mice and provides a framework for developing a novel therapeutic approach for AD.
\end{abstract}

\section{Introduction}

Photobiomodulation (PBM), also known as low-level light therapy, is a promising therapeutic approach and has been applied to various diseases as an alternative intervention $^{1-5}$. PBM refers to the low-power light $(1-500 \mathrm{~mW})$ in the visible and near-infrared (NIR) spectra to trigger beneficial biological processes in cells and tissues, leading to physiological alterations ${ }^{6}$. Although the exact mechanisms of PBM are not yet elucidated, it is believed that NIR light can activate the chromophore, including cytochrome c oxidase and heat-gated ion channels ${ }^{7-10}$, as well as restore cellular function via multi-level mechanisms. PBM

\footnotetext{
Correspondence: Liang Chen (hschenliang@fudan.edu.cn) or Xiaoling Gao (shellygao1@sjtu.edu.cn) or Xunbin Wei (xwei01@sjtu.edu.cn) ${ }^{1}$ State Key Laboratory of Oncogenes and Related Genes, Shanghai Cancer Institute, Med-X Research Institute and School of Biomedical Engineering, Shanghai Jiao Tong University, Shanghai 200030, China

${ }^{2}$ Institute of Medical Technology, Peking University Health Science Center, Beijing 100191, China

Full list of author information is available at the end of the article
}

regulates the formation of reactive oxygen species and activates the transcription factors that can upregulate or downregulate expression levels of a large number of genes $^{3,11-13}$. One of the most well-known transcription factors activated by PBM is nuclear factor kappa B, which regulates the expression of over 100 genes, including proteins with antioxidant, anti-apoptotic, pro-proliferative, and pro-migratory functions ${ }^{7}$. Therefore, PBM has been considered as a possible novel non-pharmacological and non-invasive therapeutic strategy for Alzheimer's disease $(A D)^{14-19}$, for which there are currently no effective therapeutic interventions ${ }^{20}$.

$\mathrm{AD}$, the most common form of dementia, is associated with progressive impairments in memory and cognitive skills that affect daily activities throughout the disease course, ultimately leading to death ${ }^{21}$. One of the hallmark pathologies required for the diagnosis of $A D$ is plaque deposits of the $\beta$-amyloid $(A \beta)$ peptide, which can lead to neuronal loss, neuroinflammation, cognitive deficits ${ }^{22}$.

\section{(c) The Author(s) 2021}

\footnotetext{
(c) Open Access This article is licensed under a Creative Commons Attribution 4.0 International License, which permits use, sharing, adaptation, distribution and reproduction c. in any medium or format, as long as you give appropriate credit to the original author(s) and the source, provide a link to the Creative Commons license, and indicate if changes were made. The images or other third party material in this article are included in the article's Creative Commons license, unless indicated otherwise in a credit line to the material. If material is not included in the article's Creative Commons license and your intended use is not permitted by statutory regulation or exceeds the permitted use, you will need to obtain permission directly from the copyright holder. To view a copy of this license, visit http://creativecommons.org/licenses/by/4.0/.
} 
PBM has been found to ameliorate cognitive and memory impairments in $\mathrm{AD}$ mouse models $\mathrm{s}^{3,15,16}$ and $\mathrm{AD}$ patients $^{23,24}$. There is compelling evidence suggested that PBM is capable of decreasing $A \beta$ burden in the plasma ${ }^{17}$, cerebrospinal fluid $^{17}$, and brain ${ }^{19}$ in AD models. A previous study from our group demonstrated the therapeutic effects of continuous-wave light with a wavelength of $1070 \mathrm{~nm}$ on memory abilities and $\mathrm{A} \beta$ burden in an $\mathrm{AD}$ mouse model $^{25}$. Similarly, Grillo et al. demonstrated the beneficial effects of 1070-nm light on $A \beta$ and phosphorylated tau protein levels in the brains of $\mathrm{AD}$ mice ${ }^{15}$. There is also evidence that the 1064-nm laser has beneficial neurocognitive effects in older adults ${ }^{26}$. Although a number of studies have shown the effects of continuouswave NIR light on AD, the effects of NIR light with different pulse frequencies differ. Iaccarino et al. found that $40-\mathrm{Hz}$ light flicker reduced $\mathrm{A} \beta$ and phosphorylated tau burden in the visual cortex of AD mice ${ }^{27}$. Hamblin's group studied the differences in therapeutic effects between pulsed and continuous-wave 810-nm laser treatment. The results showed that 810 -nm light pulsed at $10 \mathrm{~Hz}$ was the most effective in improving the neurological severity scores of mice with traumatic brain injury, compared with the $100-\mathrm{Hz}$ pulsed laser and continuouswave laser ${ }^{28}$. Furthermore, a number of studies demonstrated the beneficial effects of $10-\mathrm{Hz}$ pulsed light on learning and memory impairments in certain diseases, such as sleep deprivation ${ }^{29}$, traumatic brain injury ${ }^{30}$, and mild to moderately severe dementia ${ }^{23}$.

Here, we studied the effects of 1070-nm light pulsed at $10 \mathrm{~Hz}$ and $40 \mathrm{~Hz}$ on $\mathrm{APP}_{\text {swe }} / \mathrm{PS}_{\mathrm{dE} 9}$ (APP/PS1) mice, which is one of the most common animal models of $\mathrm{AD}^{31}$. We demonstrated that $1070-\mathrm{nm}$ light could improve cognitive impairment via reducing the cerebral $A \beta$ load. Engulfment by glial cells, such as microglia and astrocytes, plays a key role in the clearance of $A \beta$ deposition ${ }^{32-35}$. Microglia and astrocytes, which tightly surround plaques of the brain in $\mathrm{AD}$ patients, can engulf and degrade different forms of $A \beta^{36-40}$. Moreover, glial cells can protect the brain tissue from surrounding toxic $A \beta$ species via the formation of glial capsules around $\mathrm{A} \beta$ deposits ${ }^{41}$. Therefore, we hypothesized that 1070 -nm light could modulate glial cells to promote the clearance of $A \beta$ burden in the brains of APP/PS1 mice, leading to improvements in memory and cognitive deficits. To test this, we studied the biological responses of microglia and astrocytes triggered by $1070-n m$ light and its effects on $A \beta$ clearance and cognitive abilities. Our results showed that 1070-nm light pulsed at $10 \mathrm{~Hz}$ activated microglia rather than astrocytes to promote the degradation of $\mathrm{A} \beta$. Moreover, the $10-\mathrm{Hz}$ pulsed-light treatment reduced vessel-associated microglia and increased vessel density to further decrease $A \beta$ load in APP/PS1 mice, ultimately leading to improvements in memory ability.

\section{Results}

1070-nm light irradiation apparatus developed for APP/ PS1 mice treatments

A 1070-nm light irradiation apparatus was developed for non-invasive treatment of APP/PS1 double transgenic mice (Fig. 1a). The structure of the apparatus was described in the "Methods". During irradiation, the mice were awake and free to explore the environment in the apparatus. The pulse frequency of light used was set to $10 \mathrm{~Hz}$ (duty cycle: $50 \%$ ). We also tested the 1070 -nm light pulsed at $40 \mathrm{~Hz}$, with the same average power density and total fluence applied to compare the differences of therapeutic effects influenced only by the pulse frequency of light. Based on previous results ${ }^{25}$, the wavelength of the LED array was chosen as $1070 \pm 50 \mathrm{~nm}$, and the average power density was $25 \mathrm{~mW} / \mathrm{cm}^{2}$. The transmittances of incident 1070-nm light were summarized in Fig. 1a. No differences were found in the transmittance between different light pulse frequencies. Meanwhile, these results showed that approximately $8 \%$ of the 1070-nm light incident power penetrated through the scalp and $4.3 \%$ of that power penetrated through the scalp and skull combined. Thus, the data demonstrated that irradiance at the brain was approximately $2-4 \mathrm{~mW} / \mathrm{cm}^{2}$ when the incident irradiance was $25 \mathrm{~mW} / \mathrm{cm}^{2}$.

\section{0-nm light rescues cognitive impairment in APP/PS1 mice}

The experimental schematic was described as Fig. 1b. To test the effects of 1070-nm light on the APP/PS1 mice, mice were irradiated for 60 continuous days. During the final two weeks of irradiation, we conducted Morris water maze (MWM) and novel object recognition (NOR) tests to analyze the spatial learning and memory abilities of mice at 6 months old (6M) and 12 months old (12M). The discrimination index, which estimates the recognition ability of a novel object, was measured to determine short-term memory impairment. For $12 \mathrm{M}$ mice, the discrimination index decreased significantly in the APP/PS1 mice without irradiation (AD group) compared with the wild-type (WT) mice, and this was successfully rescued by administration of 1070-nm light pulsed at $10 \mathrm{~Hz}$ (Fig. 1c). The group of APP/PS1 mice with $10-\mathrm{Hz}$ pulsed-light irradiation $(\mathrm{AD}+10 \mathrm{~Hz})$ performed similarly to WT mice, while $40-\mathrm{Hz}$ pulsed light failed to alleviate the short-term memory deficits observed in APP/PS1 mice (Fig. 1c). However, the average velocity and total distance traveled did not differ significantly (Fig. 1d, e) among groups during the NOR test, which indicated that the higher discrimination index was not due to general differences in activities.

We next applied the MWM to test the spatial learning and memory of mice after 1070-nm light irradiation. Escape latencies in all groups showed a progressive 
a

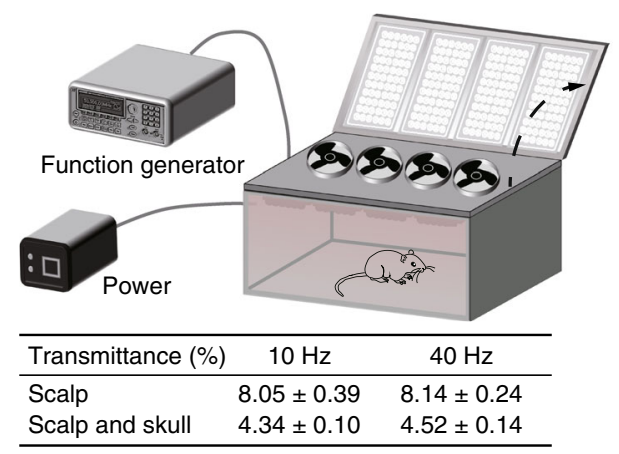

b

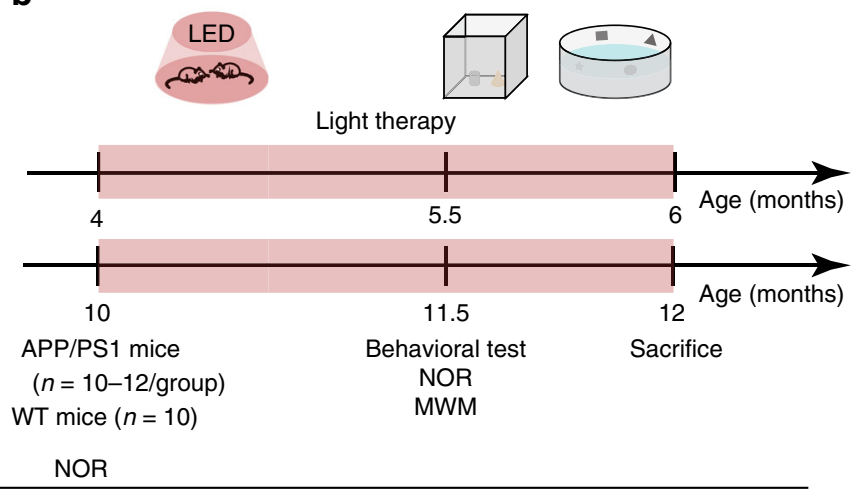

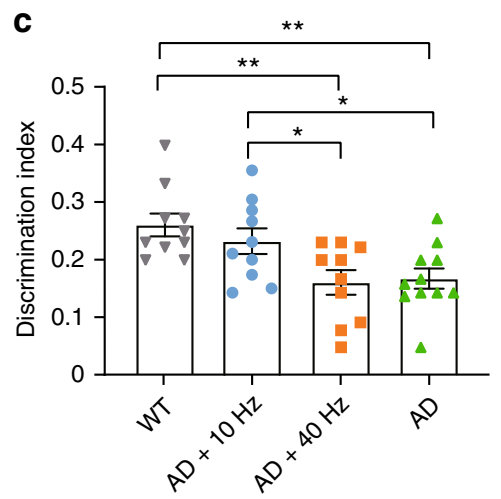

d

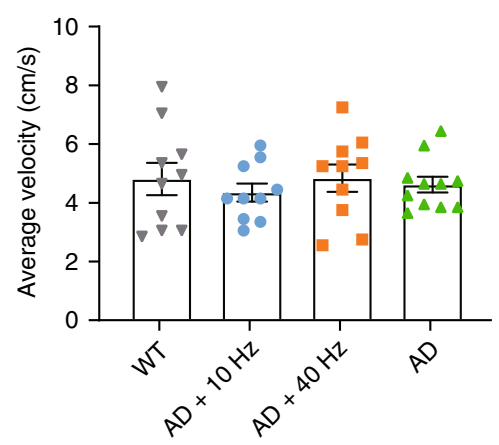

e

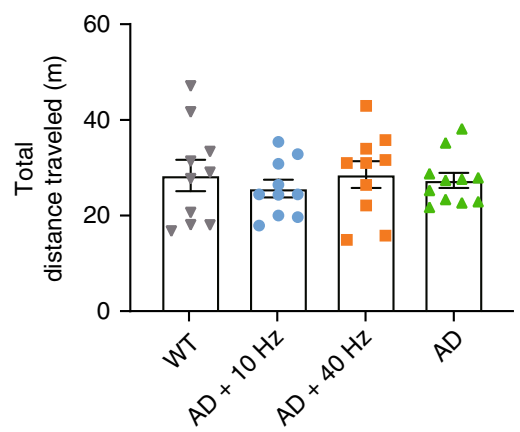

MWM
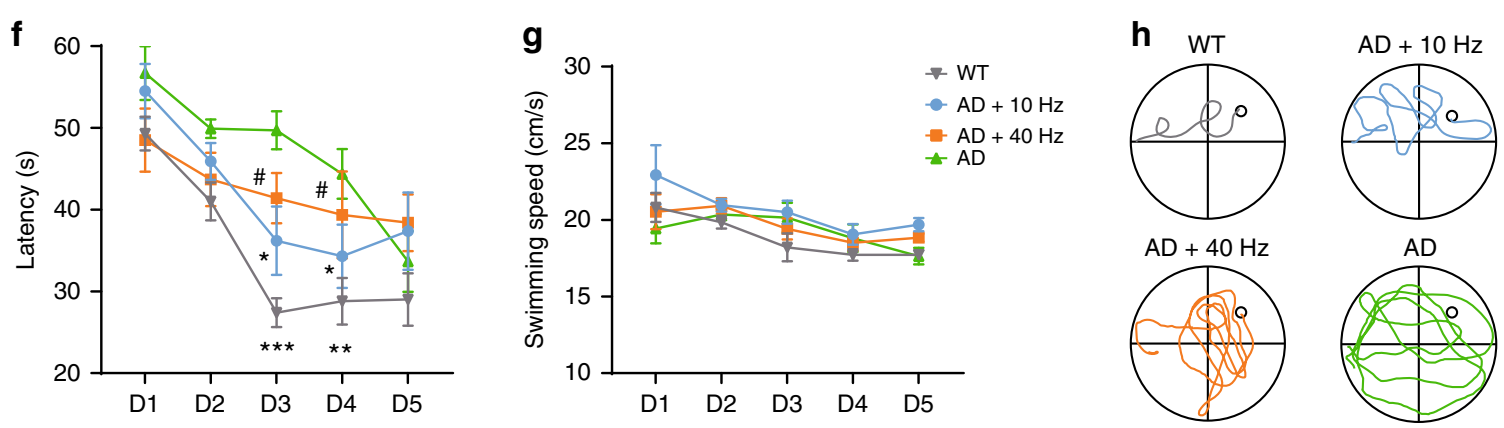

Fig. 1 1070-nm light rescues cognitive impairment in APP/PS1 mice. a The 1070-nm light irradiation apparatus and the transmittances of 1070$\mathrm{nm}$ light. $\mathbf{b}$ Experimental schematic of 1070-nm light irradiation and behavior tests. $\mathbf{c}-\mathbf{e}$ The discrimination index (c), average velocity (d) and total distance traveled (e) in the four groups during the NOR test. Data in (c)-(e) are mean \pm s.e.m., $n=10-11$ per group, ${ }^{*} p<0.05,{ }^{* *} p<0.01$. f-h Escape latency $(\mathbf{f})$, swimming speed $(\mathbf{g})$ and typical swimming path on day $4(\mathbf{h})$ of mice during the MWM. Data in $(\mathbf{f}),(\mathbf{g})$ are mean \pm s.e.m., $n=8-9$ per group. Statistically significant differences between $W T$ and $A D+40 \mathrm{~Hz}$ groups are indicated by the pound sign: \# $P<0.05$. Statistically significant differences between $A D$ group and the rest of groups are indicated by asterisks: ${ }^{*} p<0.05,{ }^{* *} p<0.01,{ }^{* * *} p<0.001$

decline during training (Fig. 1f), and this decline was less significant as the training days progressed in the $\mathrm{AD}$ group and APP/PS1 mice with 40-Hz pulsed-light irradiation $(\mathrm{AD}+40 \mathrm{~Hz})$ group. Mice in the $\mathrm{AD}$ group displayed longer escape latencies at days 3 and 4 compared with the WT mice, while the 1070-nm light pulsed at $10 \mathrm{~Hz}$ significantly decreased escape latency (Fig. 1f). Moreover, the results showed that mice in the WT and $\mathrm{AD}+10 \mathrm{~Hz}$ groups performed shorter swimming paths compared with the AD group, while there were no significant differences in average swimming speed among the four groups (Fig. 1g, h). In contrast, the results during the probe test showed no difference among groups (data not shown). During the spatial reversal test, the percentage of time spent in the southwestern (SW) quadrant was measured to determine the reversal learning impairment. Similarly, 1070-nm light pulsed at $10 \mathrm{~Hz}$ significantly raised the percentage of time in the SW quadrant 
compared with the AD group (Fig. S1a, b). However, in $6 \mathrm{M}$ mice, there were no significant differences in escape latency and time in the SW quadrant among groups (Fig. S1c-f), although the latency tended toward being shorter in the $\mathrm{AD}+10 \mathrm{~Hz}$ group, compared with the $\mathrm{AD}$ group (Fig. S1c, d). Overall, these results illustrated that 1070-nm light substantially ameliorated short-term memory and spatial learning abilities of APP/PS1 mice at $12 \mathrm{M}$.

1070-nm light attenuates $A \beta$ deposition in APP/PS1 mice

$A \beta$ deposition in APP/PS1 mice induces neuronal dysfunction, ultimately leading to impairments in cognition. We thus explored whether PBM improves cognitive and memory deficits in APP/PS1 mice via reducing cerebral amyloid pathology. The hippocampus (HPC), which plays an important role in spatial memory and consolidation of short-term memory to long-term memory ${ }^{42}$, is the first brain region that suffers damage in $\mathrm{AD}^{43}$. Meanwhile, the cortex is associated with attention, memory, language and other cognitive activities. Therefore, we examined $A \beta$ burden in the cortex and HPC of $6 \mathrm{M}$ and $12 \mathrm{M}$ APP/PS1 mice through immunostaining with anti-A $\beta$ antibody (6E10) (Fig. 2a-e) and anti-A $\beta$ antibody (D54D2) (Fig. 2f-h). Immunohistochemical results of anti-A $\beta$ antibody staining (6E10) showed no significant differences in $\mathrm{A} \beta$ burden in the HPC among groups at $6 \mathrm{M}$ (Fig. 2a, b). In contrast, the total area of $A \beta$ deposition in the cortex of $6 \mathrm{M}$ mice was reduced significantly in the $\mathrm{AD}+10 \mathrm{~Hz}$ group, compared with the $\mathrm{AD}+40 \mathrm{~Hz}(42.14 \%)$ and $\mathrm{AD}$ groups (48.96\%), respectively (Fig. 2a, d). For mice at 12M, the results revealed significantly reduced plaque area size (CA1: 45.78\%; Cortex: 26.34\%) and number (HPC: 23.50\%; CA1: 25.31\%; Cortex: $21.70 \%$ ) in the $\mathrm{AD}+10 \mathrm{~Hz}$ group, versus the $\mathrm{AD}$ group (Fig. $2 \mathrm{a}-\mathrm{c}, \mathrm{e}$ ). Mice in the $\mathrm{AD}$ $+40 \mathrm{~Hz}$ group also showed a decrease in the plaque number (HPC: 19.74\%; CA1: 26.54\%; Cortex: 11.98\%), compared with the AD group (Fig. 2a-c, e).

Similarly, immunofluorescence with anti-A $\beta$ antibody (D54D2) revealed a significant decrease in the plaque number by $43.53 \%$ and area by $33.74 \%$ in the cortex of $6 \mathrm{M}$ mice in the $\mathrm{AD}+10 \mathrm{~Hz}$ group compared with the $\mathrm{AD}$ group (Fig. 2f, g). A non-significant decrease was observed between the $\mathrm{AD}+40 \mathrm{~Hz}$ (area: $21.82 \%$; number: $31.85 \%$ ) and $\mathrm{AD}$ groups (Fig. 2f, g). However, the total area or number of $A \beta$ deposition in the HPC showed no differences among groups (Fig. S2a, b). In mice at $12 \mathrm{M}$, the results also showed that $1070-\mathrm{nm}$ light pulsed at $10 \mathrm{~Hz}$ significantly decreased $\mathrm{A} \beta$ burden in the cortex by $21.23 \%$, while no differences were found in the HPC or CA1 region, versus the AD group (Fig. 2f, h; Fig. S2a, c, d). Meanwhile, the number and area of plaques in the cortex, HPC or CA1 region did not differ significantly between the $A D+40 \mathrm{~Hz}$ and $A D$ groups (Fig. 2f, h; Fig. S2a, c, d).
To determine if there was a relationship between the performance of behavior tests and $A \beta$ level, we also performed Pearson correlation coefficient analysis. The levels of $\mathrm{A} \beta$ in the cortex of mice both at $6 \mathrm{M}$ and $12 \mathrm{M}$ were positively correlated with latency (Fig. $2 \mathrm{i}, \mathrm{j}$ ). Meanwhile, there was a negative correlation between $A \beta$ burden in the cortex of mice at $12 \mathrm{M}$ and the discrimination index during the NOR test (Fig. 2k). Taken together, these results demonstrated that $1070-\mathrm{nm}$ light could reduce $A \beta$ load in the cortex of APP/PS1 mice to improve its memory and cognitive abilities. Moreover, treating APP/ PS1 mice with 1070-nm light in the earlier phase of AD tended to reduce more $A \beta$ deposition.

\section{0-nm light reduces $A \beta$ burden via eliciting microglia response in the cortex}

Engulfing and degrading $A \beta$ protein via glial cell activity (e.g., astrocytes and microglia) is a main pathway for $A \beta$ clearance in the $\mathrm{AD}^{32-35}$. Thus, the effects of 1070-nm light on astrocytes and microglia were tested to explore if light-activated microglia or astrocytes would promote $A \beta$ clearance. Activated microglia were identified by a deramified phenotype which referred to retracted processes, enlarged cell bodies, and the high capacity for phagocytosis $^{44-46}$. We thus analyzed the morphological alterations in microglia. For mice at $6 \mathrm{M}$, we observed light-modified morphological features in microglia. Microglia were significantly reduced in number $(24.13 \%)$ and length (26.59\%) of branches after $10-\mathrm{Hz}$ pulsed-light treatment, compared with the AD group (Fig. 3a-d). Likewise, the microglial volume was substantially increased by $31.99 \%$ in mice of the $A D+10 \mathrm{~Hz}$ group, versus the $A D$ group (Fig. 3e). Nevertheless, $40-\mathrm{Hz}$ pulsed light failed to change the morphology of microglia in the cortex (Fig. 3a-e). For mice at $12 \mathrm{M}$, the morphology of microglia did not differ among groups (Fig. S3a). However, the length and number of branches in mice at $12 \mathrm{M}$ decreased compared to 6M mice (Fig. 3c, d, Fig. S3a), which indicated that most microglia were activated in mice at $12 \mathrm{M}$. There were also no differences in the ionized calcium-binding adapter molecule 1 (Iba1) burden among groups at $6 \mathrm{M}$ or $12 \mathrm{M}$, further suggesting that $1070-\mathrm{nm}$ light changed the morphology rather than the amount of microglia (Fig. S3b, c).

Activated microglia have a high capacity for engulfment ${ }^{46,47}$. The effects shown above regarding 1070-nm light and microglia activation led us to explore the colocalization between $A \beta$ and microglia. Indeed, the $A D$ $+10 \mathrm{~Hz}$ group displayed an increase in the colocalization between microglia and $A \beta$ in the cortex of mice at $12 \mathrm{M}$, while no significant increase was observed in the $A D$ $+40 \mathrm{~Hz}$ group (Fig. 3f, g, i). For mice at $6 \mathrm{M}$, there were no differences in the colocalization between $A \beta$ plaques and microglia in the $\mathrm{AD}+10 \mathrm{~Hz}$ group, versus the $\mathrm{AD}$ mice (Fig. S3d). Furthermore, the results revealed that the 


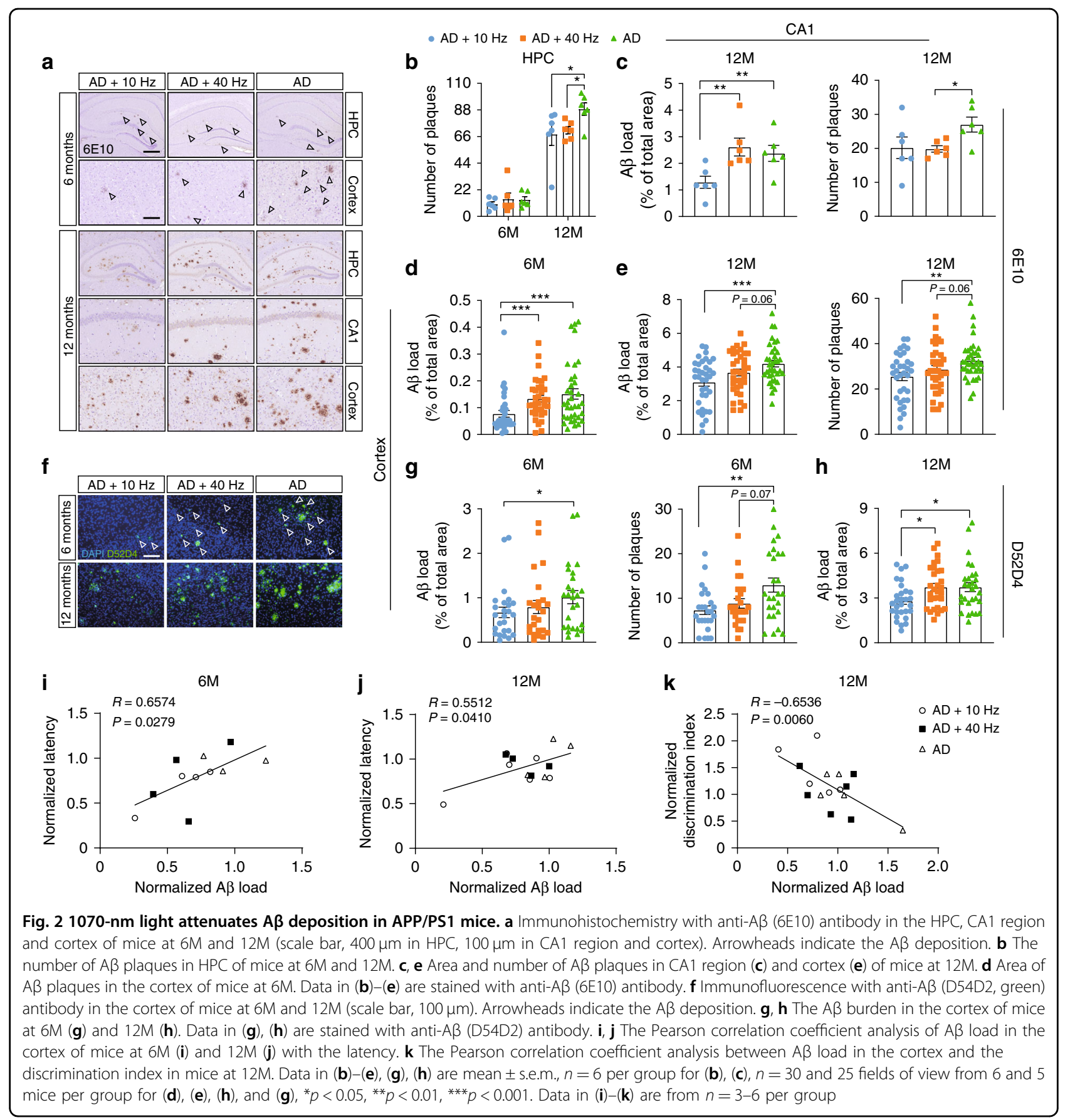

number and length of branches were positively correlated with $A \beta$ load in the cortex of $6 \mathrm{M}$ mice, respectively (Fig. $3 \mathrm{~h}, \mathrm{j}$ ). There was also a negative correlation between the percentage of $A \beta$ within microglia and $A \beta$ load in the cortex of $12 \mathrm{M}$ mice (Fig. 3k).

In addition to microglia, astrocytes also uptake and degrade different forms of $A \beta$. We thus assessed the effects of 1070-nm light on astrocytes in AD mice. For mice at $6 \mathrm{M}$, glial fibrillary acidic protein (GFAP)-positive areas in the cortex were reduced by $23.99 \%$ in the $\mathrm{AD}$
$+40 \mathrm{~Hz}$ group, while $10-\mathrm{Hz}$ pulsed light failed to reduce GFAP-positive cells, compared with the AD group (Fig. S4a, b). Mice in the AD $+40 \mathrm{~Hz}$ group showed a $16.31 \%$ increase in the colocalization between astrocytes and $A \beta$ (Fig. S4a, b). However, no significant differences in GFAPpositive areas or colocalization of astrocytes with $A \beta$ in the cortex were found in $12 \mathrm{M}$ mice (Fig. S4a, c). Moreover, the correlation analysis showed that the 1070-nm light-induced alterations in mice at $6 \mathrm{M}$ were not correlated with $A \beta$ load or latency (Fig. S4d-f). Overall, these 
a

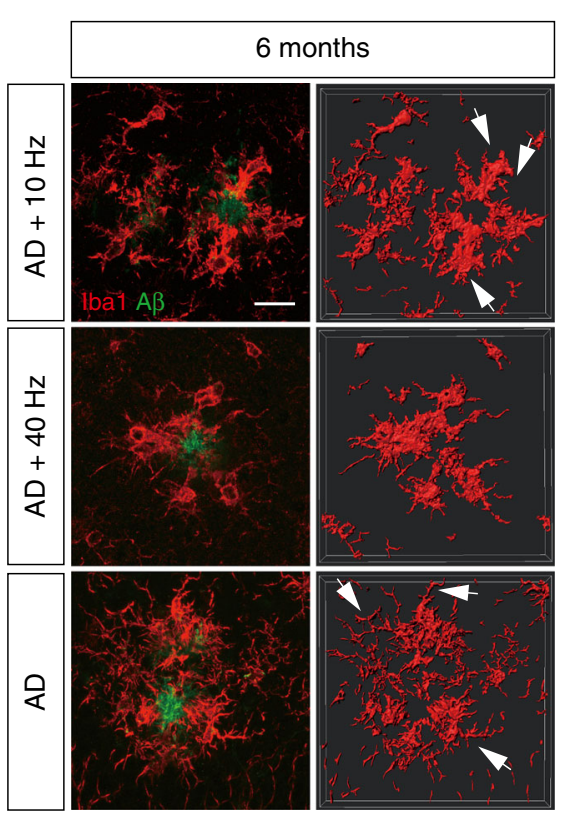

b
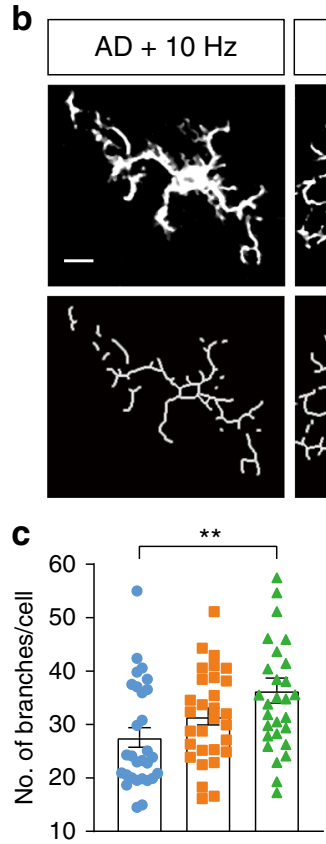
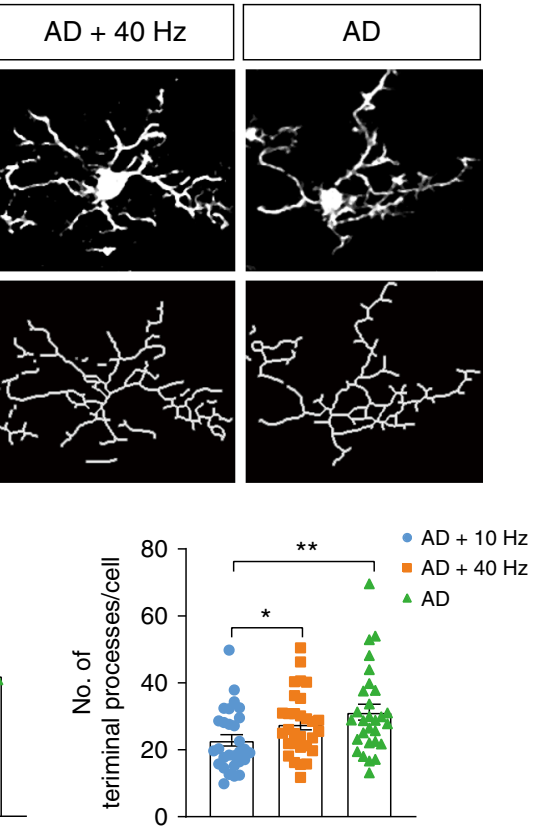

d
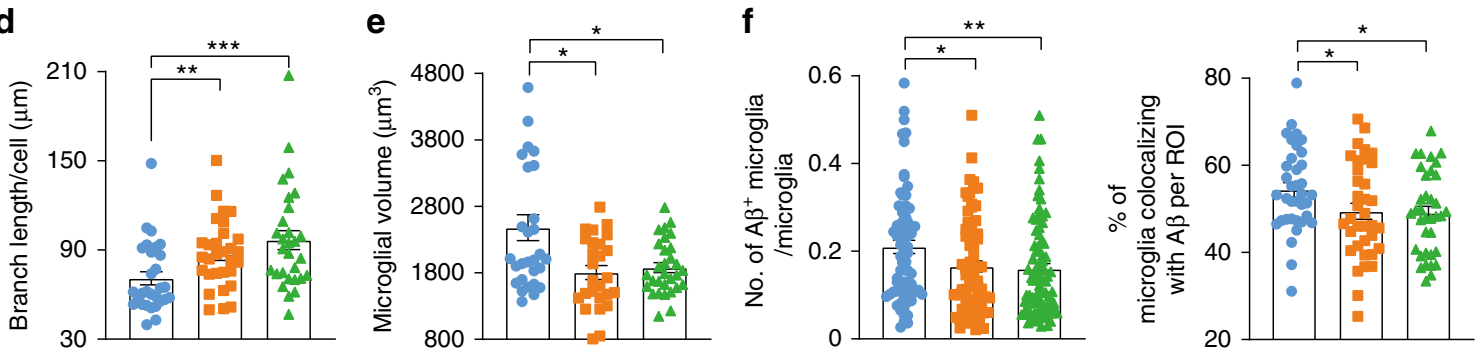

g
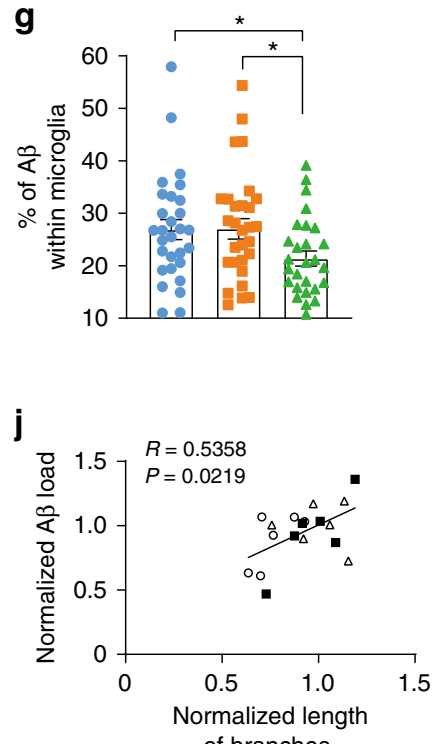

of branches

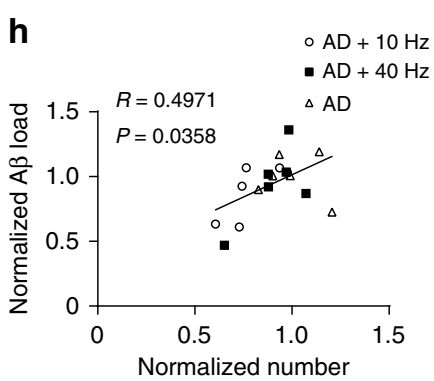

of branches

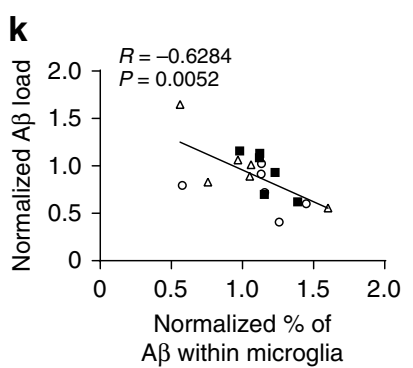

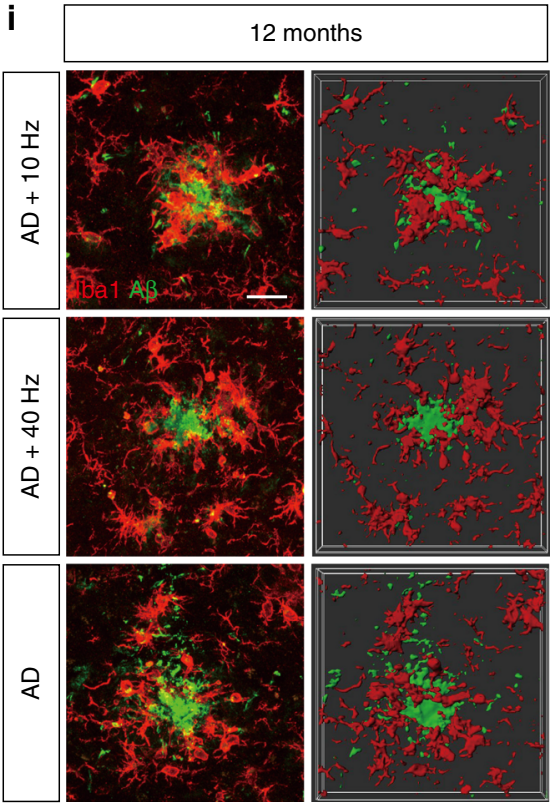

Fig. 3 (See legend on next page.) 
(see figure on previous page)

Fig. 3 1070-nm light reduces $\mathbf{A} \boldsymbol{\beta}$ burden via eliciting microglia response in the cortex. $\mathbf{a}, \mathbf{i}$ Immunofluorescence with anti-lba1 (red) and anti$A \beta$ (D54D2, green) antibodies in the cortex of APP/PS1 mice at 6M (a) and 12M (i) (scale bar, $20 \mu \mathrm{m}$ ). Arrowheads indicate the branches of microglia. b The topological skeletonized images of typical microglia from the three groups. Scale bar, $10 \mu \mathrm{m}$. c-e Number of branches and terminal processes per cell (c), branch length per cell $(\mathbf{d})$, and microglial volume (e) in mice at $6 \mathrm{M}$. $\mathbf{f}, \mathbf{g}$ The colocalization between microglia and $A \beta$ deposition in mice at $12 \mathrm{M} . \mathbf{h}, \mathbf{j}$ The Pearson correlation coefficient analysis of $A \beta$ load with the number $(\mathbf{h})$ and length (j) of branches in mice at $6 \mathrm{M}$. $\mathbf{k}$ The Pearson correlation coefficient analysis between the $A \beta$ load and percentage of $A \beta$ within microglia in mice at $12 \mathrm{M}$. Data in $(\mathbf{c})-(\mathbf{g})$ are mean \pm s.e.m., $n=30$ fields of view from 6 mice per group for $(\mathbf{c})-(\mathbf{g}),{ }^{*} p<0.05,{ }^{* *} p<0.01,{ }^{* * *} p<0.001$. Data in $(\mathbf{h}),(\mathbf{j})$, (k) are from $n=5-6$ per group

results indicated that 1070 -nm light-activated microglia rather than astrocytes to promote the degradation of $\mathrm{A} \beta$.

\section{0-nm light decreases M1-like microglia surrounding the vessels in the cortex}

Activated microglia have two different polarization states. M1-like microglia, which produce pro-inflammatory factors, are increased in $\mathrm{AD}$ patients. It leads to oxidative stress and cognitive deficits ${ }^{48}$. In contrast, M2-like microglia exert a neuroprotective effect and induce tissue repair $^{49,50}$. To assess whether 1070 -nm light would change M1/M2 polarization to decrease the impairment induced by M1-like microglia, we analyzed M1 and M2 markers, respectively. Results showed that the fluorescence intensity of the M1 marker, Gyclooxygenase-2 (COX-2), had no differences among the three groups (Fig. 4a, c). In contrast, the percentage of $\mathrm{COX}-2^{+}$microglia was decreased by $16.30 \%$ in the $\mathrm{AD}+10 \mathrm{~Hz}$ group at $6 \mathrm{M}$, compared with the AD group (Fig. 4a, d). For mice at 12M, 1070-nm light pulsed at $10 \mathrm{~Hz}$ significantly reduced the fluorescence intensity of COX-2 in the cortex, compared with the $\mathrm{AD}$ $(16.58 \%)$ and $\mathrm{AD}+40 \mathrm{~Hz}$ groups (24.05\%), indicating that M1-like microglia were reduced after $10-\mathrm{Hz}$ pulsed-light irradiation (Fig. S5). In contrast, few microglia with M2 marker CD163 were found among the three groups (Fig. S6), indicating that there were not many M2-like microglia in the $\mathrm{AD}$ mice.

Perivascular microglia, especially perivascular M1-like microglia, stimulate endothelial cells and pericytes of the blood-brain barrier, as well as decrease tight junction proteins, ultimately leading to cerebrovascular dysfunction in $\mathrm{AD}^{51}$. We thus explored if 1070 -nm light would reduce perivascular M1-like microglia to increase vessel density. To test this, we analyzed the perivascular microglia. The results showed that there was a decrease in the number of perivascular microglia in the 1070-nm light groups at $6 \mathrm{M}$ (Fig. 4b, e, f). Moreover, there was also a decrease in M1-like microglia surrounding vessels in the $\mathrm{AD}+10 \mathrm{~Hz}$ group at $6 \mathrm{M}(20.35 \%)$, while $40-\mathrm{Hz}$ pulsed light failed to reduce the M1 phenotype in perivascular microglia (Fig. 4g, h). Altogether, these results indicated that $1070-\mathrm{nm}$ light pulsed at $10 \mathrm{~Hz}$ could reduce vessel-associated microglia and perivascular M1like microglia.

\section{0-nm light decreases $A \beta$ deposition via increasing cerebral vessel density}

The results in the perivascular microglia led us to investigate if 1070-nm light could rescue the decreased vessel density in APP/PS1 mice. For mice at $6 \mathrm{M}$, increased vessel density (24.26\%) and length (30.74\%) in the cortex were found in the $\mathrm{AD}+10 \mathrm{~Hz}$ group, versus the $\mathrm{AD}$ group (Fig. 5a, b). In addition, there was a slight, non-significant increase in vessel length (15.56\%) in the cortex of the AD $+40 \mathrm{~Hz}$ group (Fig. 5a, b). Furthermore, Pearson correlation coefficient analysis displayed a strong correlation of 1070$n m$ light-induced alterations of vessel with $A \beta$ load and latency in MWM (Fig. 5c, d), which indicated that 1070-nm light could also promote the clearance of $A \beta$ deposition in mice at $6 \mathrm{M}$ via amelioration of cerebral vessel impairment.

A previous study showed that high vessel endothelium growth factor (VEGF) levels in the brains of patients were positively correlated with good performance in cognitive tests $^{52}$. We thus analyzed the levels of VEGF in the cortex of mice at $6 \mathrm{M}$. The results showed that VEGF levels were increased by $38.12 \%$ in the $\mathrm{AD}+10 \mathrm{~Hz}$ group, versus the $\mathrm{AD}$ group (Fig. 5e). The extracellular signal-regulated kinase (ERK) pathway, which is a key signaling pathway that regulates a wide variety of cellular processes, can mediate the levels of VEGF ${ }^{53}$. Therefore, we examined the levels of phosphorylated ERK (p-ERK) and ERK. A significant increase in p-ERK levels was found in the $A D$ $+10 \mathrm{~Hz}(48.19 \%)$ versus the AD group, while the ERK level did not differ significantly among groups (Fig. 5f). The phosphoinositide 3-kinase (PI3K)/protein kinase B (AKT) pathway, which is an important intracellular signaling pathway in regulating the cell cycle, also mediates the levels of $\mathrm{VEGF}^{54}$. However, the results showed no differences in the levels of phosphorylated PI3K (p-PI3K), PI3K, phosphorylated AKT (p-AKT) or AKT among groups at $6 \mathrm{M}$ (Fig. 5g, h). Overall, 1070-nm light could modulate the VEGF levels to increase cerebral vessel density in the cortex of APP/PS1 mice at early pathological stage. Moreover, increased vessel density in the 1070-nm light group could promote the clearance of $A \beta$.

\section{Discussion}

In the present study, the main findings have demonstrated the therapeutic effects of 1070-nm light on the 


\section{$\mathbf{a}$}
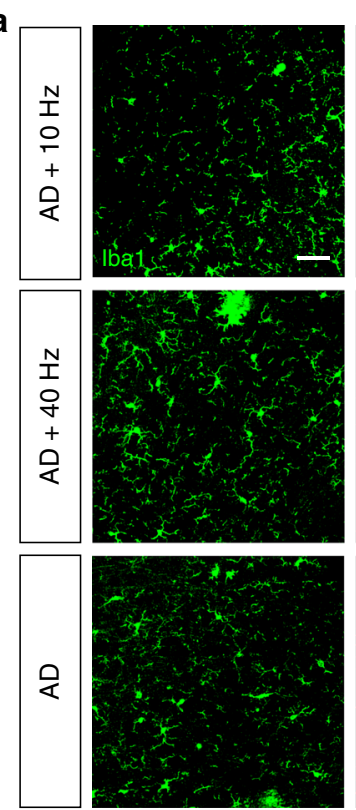

C
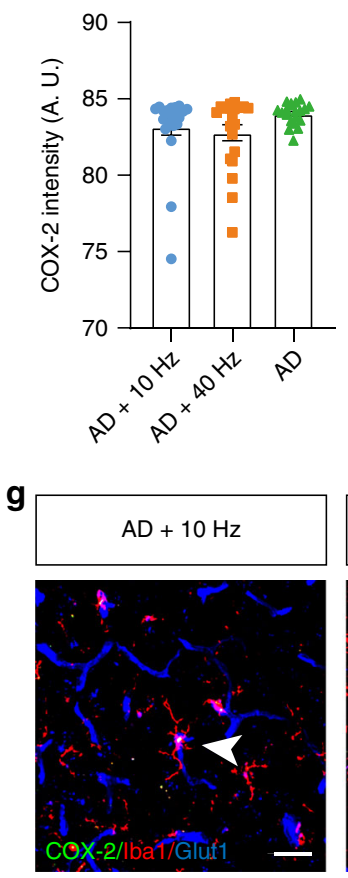
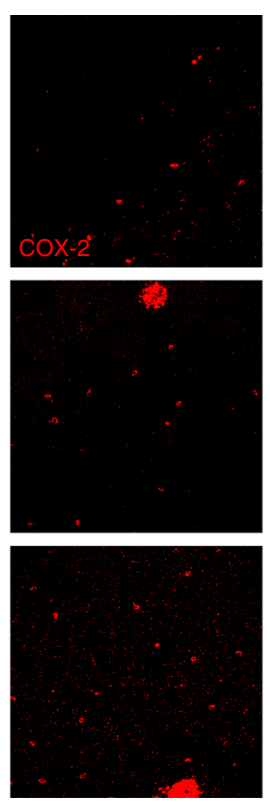

d
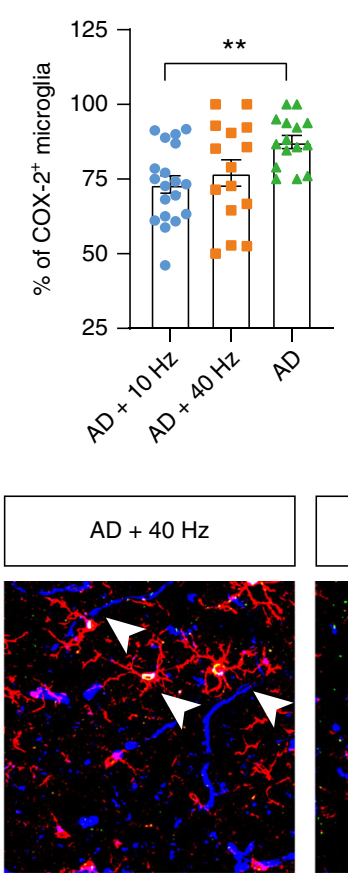

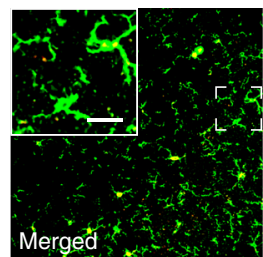

b
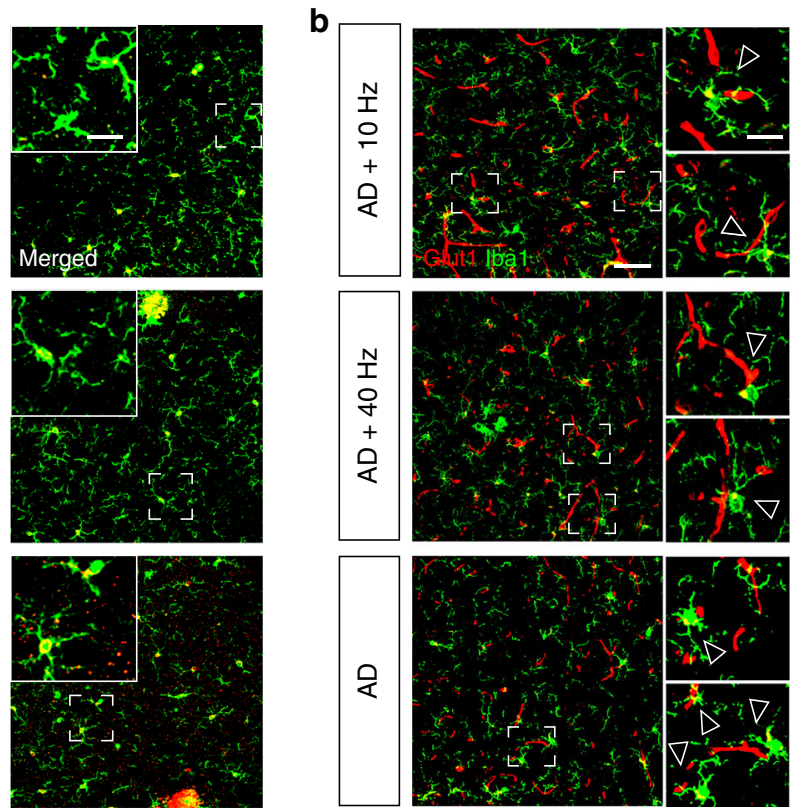

e
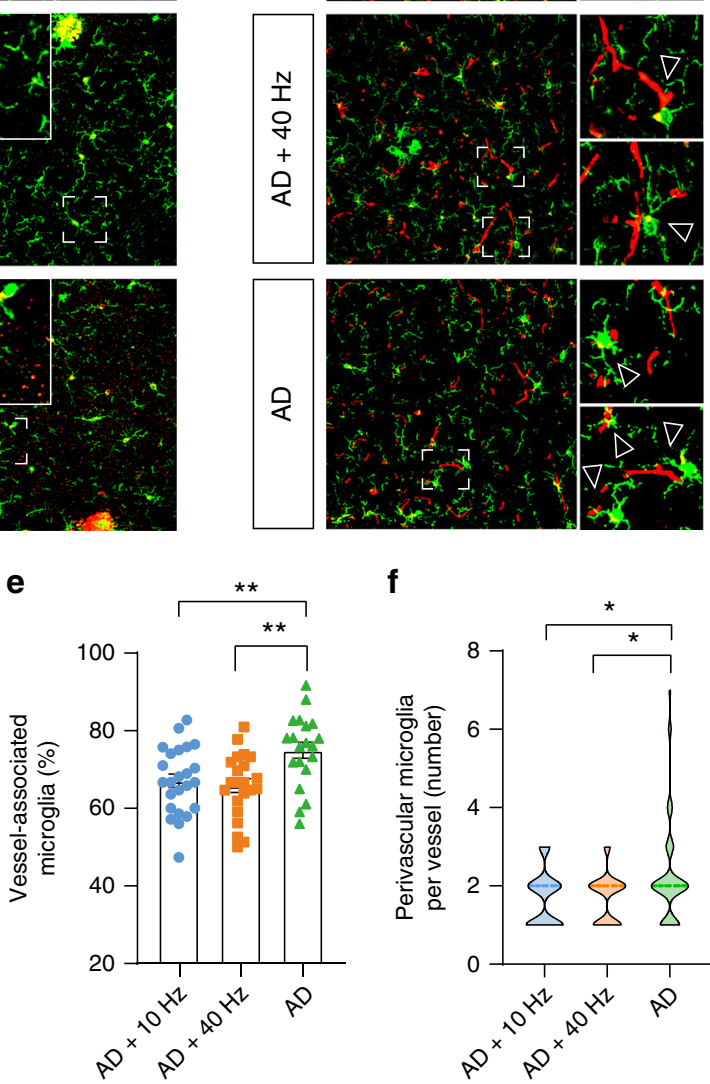

$\mathbf{f}$

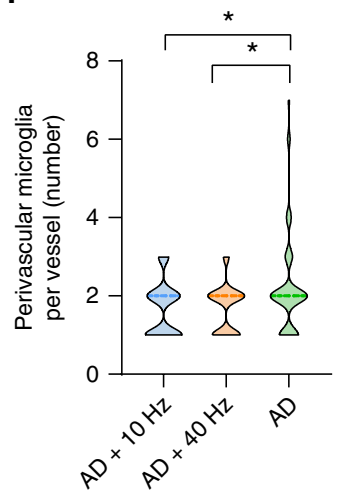

h
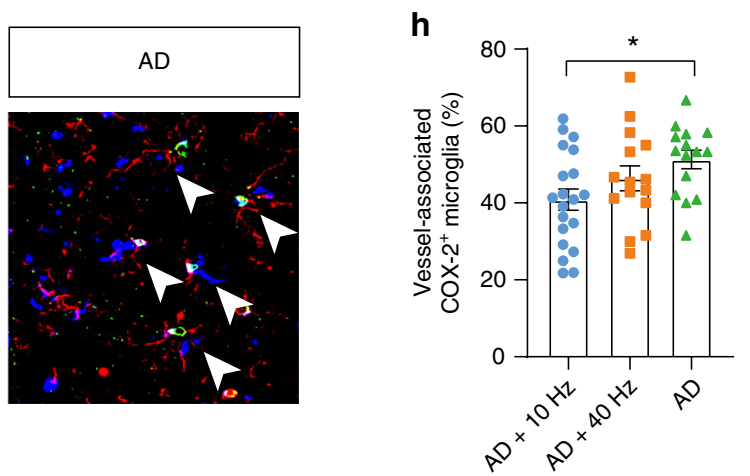

Fig. 4 1070-nm light decreases M1-like microglia surrounding the vessels in the cortex of APP/PS1 mice at 6M. a Immunofluorescence with anti-Iba1 (green) and anti-COX-2 (red) antibodies in the cortex of APP/PS1 mice at 6M (scale bar, $50 \mu \mathrm{m}$ ). The magnified images show the typical microglia expressing COX-2 (scale bar, $20 \mu \mathrm{m}$ ). b Immunofluorescence with anti-lba1 (green) and anti-Glut1 (red) antibodies in the cortex of mice at $6 \mathrm{M}$ (scale bar, $50 \mu \mathrm{m})$. Arrowheads and the magnified images show the typical blood vessels with associated microglia (scale bar, $20 \mu \mathrm{m})$. $\mathbf{c}$, $\mathbf{d}$ COX-2 fluorescence intensity (c) and the percentage of COX-2 positive microglia (d) in mice at $6 \mathrm{M}$. e, $\mathbf{f}$ The percentage of vessel-associated microglia (e) and the number of perivascular microglia per vessel (f) in mice at $6 \mathrm{M}$. $\mathbf{g}$ Immunofluorescence with anti-Iba1 (red), anti-COX-2 (green), and anti-Glut1 (blue) antibodies in the cortex of mice at $6 \mathrm{M}$ (scale bar, $25 \mu \mathrm{m}$ ). Arrowheads indicate contact between $\mathrm{COX}-2^{+}$microglia and blood vessels. $\mathbf{h}$ The percentage of $\mathrm{COX}_{-} 2^{+}$microglia surrounding vessels in the cortex of mice at $6 \mathrm{M}$. Data in $(\mathbf{c})-(\mathbf{f})$ and $(\mathbf{h})$ are mean \pm s.e.m., $n=15-20$ fields of view from 3-4 mice per group for (c)-(e) and (h), $n=60-63$ vessels from 4 mice per group for $(\mathbf{f}),{ }^{*} p<0.05,{ }^{*} p<0.01$ 
a
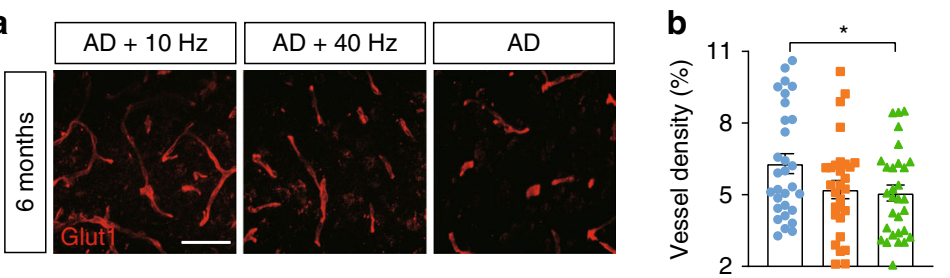

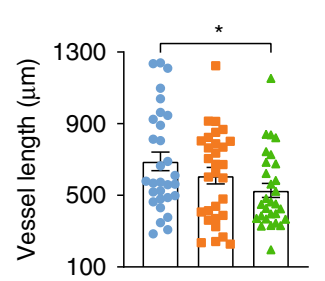

- $A D+10 \mathrm{~Hz}$

- $\mathrm{AD}+40 \mathrm{~Hz}$

$\triangle \mathrm{AD}$
C

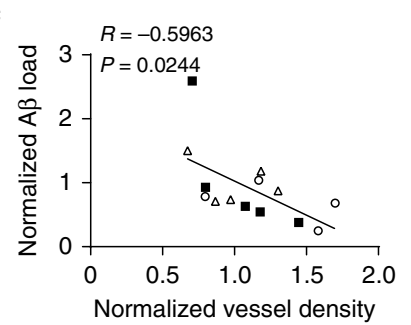

e

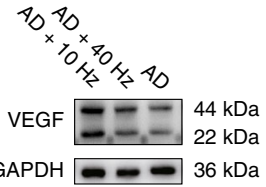

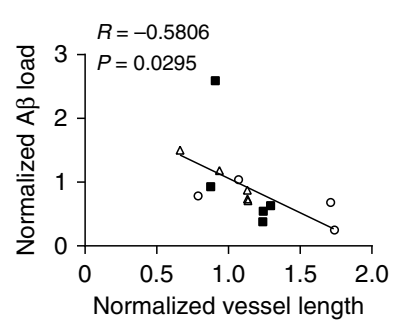

\section{d}

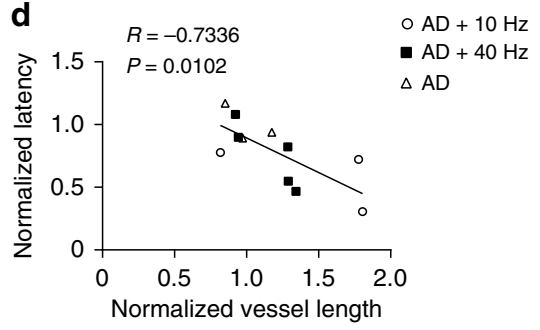

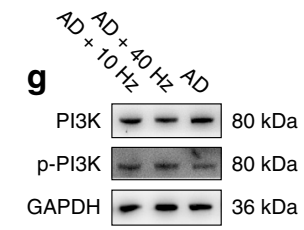

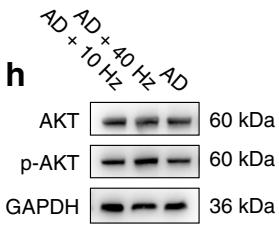

- $\mathrm{AD}+10 \mathrm{~Hz} \quad \square \mathrm{AD}+40 \mathrm{~Hz} \quad \triangle \mathrm{AD}$
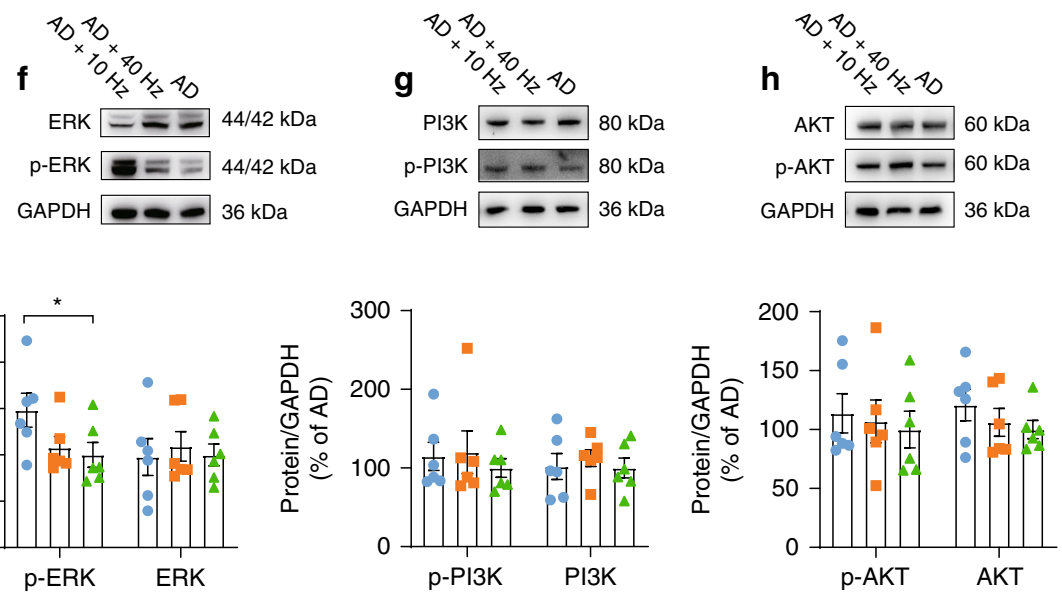

Fig. 5 1070-nm light decreases $\mathbf{A} \boldsymbol{\beta}$ deposition via increasing cerebral vessel density. a Immunofluorescence with anti-Glut1 antibody in the cortex of mice at $6 \mathrm{M}$ (scale bar, $50 \mu \mathrm{m}$ ). $\mathbf{b}$ Vessel density and length in the cortex of mice at $6 \mathrm{M}$. $\mathbf{c}$, $\mathbf{d}$ The Pearson correlation coefficient analysis of vessel density and length with $A \beta$ load (c) and latency (d) in mice at $6 \mathrm{M}$. e-h Western blot analysis for VEGF (e), ERK, p-ERK (f), PI3K, p-PI3K (g), AKT, p-AKT (h) and GAPDH in the cortex of mice at $6 \mathrm{M}$. Data in (b), (e)-(h) are mean \pm s.e.m., $n=30$ fields of view from 6 mice per group for $(\mathbf{b}), n=6$ per group for $(\mathbf{e})-(\mathbf{h}),{ }^{*} p<0.05$. Data in $(\mathbf{c})$, (d) are from $n=3-5$ per group

memory and cognition impairments, as well as on $\mathrm{A} \beta$ clearance in APP/PS1 mice. Our results show that 1070$\mathrm{nm}$ light pulsed at $10 \mathrm{~Hz}$ triggers microglia responses with alteration in morphology and increase in its colocalization with $A \beta$, instead of triggering astrocyte responses. The responses of microglia to $1070-\mathrm{nm}$ light are negatively correlated with the level of $A \beta$, suggesting that 1070-nm light pulsed at $10 \mathrm{~Hz}$ can reduce the $A \beta$ burden via eliciting microglia activation and recruiting microglia to $A \beta$ deposition. The perivascular microglia, especially M1-like microglia, decrease after $10-\mathrm{Hz}$ pulsed-light treatment, while an increase in cerebral vessel density is found in AD $+10 \mathrm{~Hz}$ group. Moreover, vessel density is positively correlated with clearance of $\mathrm{A} \beta$ deposition, suggesting that $1070-\mathrm{nm}$ light pulsed at $10 \mathrm{~Hz}$ can also reduce $\mathrm{A} \beta$ burden via increasing cerebral vessel density. Overall, our findings suggest that $1070-\mathrm{nm}$ light pulsed at $10 \mathrm{~Hz}$ can reduce cerebral $A \beta$ levels and thus improve memory and cognition abilities through activation of microglia and promotion of angiogenesis. Therefore, 1070-nm light may be a practical and promising novel therapeutic strategy for treating AD.

Iaccarino et al. demonstrated that $40-\mathrm{Hz}$ visual stimulation increased the power of gamma oscillation to reduce $\mathrm{A} \beta$ burden in the visual cortex via activation of microglia and changes in the morphology of microglia in 5xFAD mice $^{27}$. Moreover, they found that gamma entrainment using sensory stimulus (GENUS) could improve the cognitive and memory impairments in $5 x F A D$ mice and reduce $A \beta$ load in the $\mathrm{HPC}^{55}$. Here, our results indicated that $1070-\mathrm{nm}$ light pulsed at $10 \mathrm{~Hz}$ was more effective in treating AD mice compared with the $40-\mathrm{Hz}$ pulsed light. The $10-\mathrm{Hz}$ pulsed light used in this study can induce alterations in the widespread cortex, while the effects of 
gamma visual stimulation focus on the visual cortex. Meanwhile, the $10-\mathrm{Hz}$ pulsed light used here is invisible so that it cannot trigger GENUS (responding to visible light), further suggesting that the mechanisms underlying the beneficial effects of $10-\mathrm{Hz}$ pulsed-light stimulation and GENUS are different. One explanation as to why $1070-\mathrm{nm}$ light pulsed at $10 \mathrm{~Hz}$ is more effective for treating $\mathrm{AD}$ is that its period $(100 \mathrm{~ms})$ is similar to the duration of some biological activities involved in the light stimulation ${ }^{28}$. Transient receptor potential (TRP) channels, the calcium ion channels, is reported to serve as photoreceptors and are responsible for some of the mechanisms of $\mathrm{PBM}^{56-59}$. Moreover, the half-life of the burst length of TRPV1 with activation of $0.25-\mu \mathrm{M}$ capsaicin is $94.5 \pm 30 \mathrm{~ms}$, suggesting that $10-\mathrm{Hz}$ pulsed-light stimulation may activate related ion channels to trigger a series of biological responses. However, more studies are necessary to explore mechanisms of the pulse frequencydependent effects of 1070-nm light treatment on AD.

Previous studies have shown a reduction of $\mathrm{A} \beta$ burden in AD mice after 1070-nm light treatment ${ }^{17,19}$. However, few studies have focused on its effects at different stages of pathological progression. In this study, our results showed a trend that irradiating mice in the early pathological stage of $\mathrm{AD}$ was more effective to treat $\mathrm{AD}$ than those in the late stage (Fig. 2d, e, g, h). Das et al. found that treating APP Tg2576 with $\gamma$-secretase inhibitor during the pre-deposition "seeding" phase (4-7M) had the most significant efficacy on the reduction in $A \beta$ burden. In contrast, treatment during the exponential phase of deposition $(7-10 \mathrm{M}$ or $12-15 \mathrm{M})$ showed progressively decreasing efficacy of treatment ${ }^{60}$. Our findings further suggested the more significant impact of 1070-nm light treatment occurred during the early stage of $\mathrm{AD}$ compared with the later stage. The 1070-nm light might reduce more $A \beta$ plaques at the pre-deposition phase by attenuating the seeding of $A \beta$, which was critical to the development of $A \beta$ deposition. In contrast, the effects of 1070-nm light on $A \beta$ load in the later stage were not sufficient, considering the rapid growth rate of $A \beta$ plaques. Therefore, 1070-nm light had less significant efficacy on the $A \beta$ clearance in the late stage of $A D$.

The morphological alteration of microglia, which indicates microglia activation, appears at the early stage of $\mathrm{AD}^{61}$. Activated microglia can reduce the $\mathrm{A} \beta$ burden at the early stage of $\mathrm{AD}$ via increasing its phagocytosis and degradation ${ }^{62,63}$. Similarly, we have found that 1070-nm light promotes the activation of microglia to reduce the $A \beta$ burden at the early stage of $A D$ (Fig. 3a-e, $h, j)$. Although a large number of microglia are activated with the pathological progression ${ }^{61,64}$, microglia show inefficient phagocytic and clearing ability of $A \beta^{65,66}$ at the late stage of AD. Our results indicate that 1070-nm light promotes the phagocytic ability of microglia at the late stage of AD (Fig. 3f, g, i, k). Therefore, 1070-nm light is likely to enhance the innate responses of microglia to $A \beta$ clearance which change with the pathological progression of $\mathrm{AD}$.

One particular study demonstrated that $800-\mathrm{nm}$ laser increased the M2-like microglia and decreased M1-like microglia, resulting in improvements in spinal cord injury $^{67}$. Leden et al. also showed that $808-\mathrm{nm}$ light induced a dose-dependent alteration in M1/M2 polarization and promoted neurite growth ${ }^{68}$. Similarly, our results displayed that M1-like microglia declined after $10-\mathrm{Hz}$ pulsed-light irradiation. Microglia were reported to localize to perivascular $A \beta$ depositions ${ }^{69}$. These plaqueassociated microglia express inflammatory factors, such as interleukin- $1 \beta$ and tumor necrosis factor- $\alpha$ and mostly exhibit an M1-like phenotype ${ }^{70}$. Similarly, we found that increased perivascular microglia in $\mathrm{AD}$ mice and they were reduced after 1070-nm light treatment. The M1-like microglia around vessels were also decreased by $1070-\mathrm{nm}$ light pulsed at $10 \mathrm{~Hz}$. These perivascular microglia, especially perivascular M1-like microglia, promoted cerebrovascular dysfunction, leading to the deficiency in $A \beta$ clearance and cerebral metabolism. Indeed, we observed an increase in cerebral vessel density after $10-\mathrm{Hz}$ pulsedlight irradiation, suggesting that 1070 -nm light pulsed at $10 \mathrm{~Hz}$ may promote vessel density via microglia modulation. In addition, our results demonstrated that 1070-nm light pulsed at $10 \mathrm{~Hz}$ increased VEGF levels and activated VEGF-related cellular pathways. A number of studies also found the upregulation of VEGF levels via the ERK pathway after light irradiation ${ }^{4,71,72}$. Therefore, this indicated another possible mechanism underlying the increase of vessel density, which was that the 1070-nm light pulsed at $10 \mathrm{~Hz}$ upregulated VEGF levels to promote angiogenesis in the cortex.

In conclusion, our results demonstrate the effects of 1070-nm light on microglia modulation and cerebral vessels during different phases of $\mathrm{AD}$ and provide valuable insight into the mechanisms of 1070-nm light treatment to $\mathrm{AD}$. This is beneficial for the exploration of optimal parameters when administering PBM as well as the development of a promising and novel therapeutic approach for AD.

\section{Materials and methods \\ Animals}

Female $\mathrm{APP}_{\text {swe }} / \mathrm{PS}_{\mathrm{dE}}$ (APP/PS1) double transgenic mice and age-matched wild-type (WT) littermates were utilized to explore the effects after 1070-nm light treatment. APP/PS1 mice overexpressing the hAPP695swe (APP695swe) and mutant human presenilin 1(PS1-dE9) on the C57BL/6 background were obtained from Nanjing Biomedical Research Institute of Nanjing University. To explore the differences of effects after 1070-nm light 
treatment at different onset stages, $4 \mathrm{M}$ and $10 \mathrm{M}$ mice received 60-day treatment, respectively. All mice with different ages were divided into four groups $(n=10-12$ per group): one treatment group with $10-\mathrm{Hz}$ pulsed-light irradiation $(\mathrm{AD}+10 \mathrm{~Hz})$, one treatment group with $40-\mathrm{Hz}$ pulsed-light irradiation $(\mathrm{AD}+40 \mathrm{~Hz})$, one sham treatment group (AD), and one negative control group (WT). The AD group and WT group represented APP/ PS1 mice with non-irradiated and normal mice, respectively. All mice were housed under a 12/12-h light-dark cycle with food and water ad libitum. Animal care and experimental protocols were approved by the Shanghai Jiao Tong University Ethical Committee of Animal Experiments.

In the last 15 days of irradiation treatment, we tested the memory and spatial learning abilities of all mice via the NOR and MWM tests (Fig. 1b). After behavioral tests, mice were humanely sacrificed. The brains were quickly dissected and stored at $-80^{\circ} \mathrm{C}$ until ready for further analysis.

\section{Apparatus and treatment}

The apparatus consisted of a chamber and a lightemitting diode (LED) array as the lid. The average power of the LED array was $900 \mathrm{~mW}$, and the average power density and the total fluence were $25 \mathrm{~mW} / \mathrm{cm}^{2}$ and $4.5 \mathrm{~J} / \mathrm{cm}^{2}$, respectively. The light pulse frequencies were set to $10 \mathrm{~Hz}$ and $40 \mathrm{~Hz}$ (duty cycle: $50 \%$ ). The cooling fans were utilized to reduce the influence of thermal effects induced by the LED array.

For 1070-nm light treatment, mice in the $\mathrm{AD}+10 \mathrm{~Hz}$ and $\mathrm{AD}+40 \mathrm{~Hz}$ groups were placed in the $1070-\mathrm{nm}$ light device and received irradiation of 6 min per day at 7 P.M. for 60 consecutive days, with the wavelength at $1070 \pm$ $50 \mathrm{~nm}$. During treatments, mice can ambulate, explore, and rest. Mice in the AD and WT groups underwent the same procedures as the treatment groups, except that the 1070-nm light device remained off.

\section{Behavioral tests \\ Novel object recognition test}

The NOR test, which relies on a rodent's natural proclivity for exploring novelty, is a common method for examining cognition, particularly recognition memory ${ }^{73}$. We performed the NOR test on day 46 of 1070-nm light treatment to measure the recognition memory of mice. During the habituation session, mice were placed into the empty open field and allowed to freely explore for $5 \mathrm{~min}$. After a $24 \mathrm{~h}$ period, two identical non-toxic objects were placed in opposite and symmetrical corners of the arena; each mouse was released into the open field and allowed free exploration for a 10-min period. One of the previously explored objects was replaced $6 \mathrm{~h}$ later by a novel object. The mice were returned to explore the open field for an additional $10 \mathrm{~min}$ to test preference for a novel object. Object preference was analyzed by a discrimination index, calculated as follows:

$\mathrm{DI}=\left(T_{\text {novel }}-T_{\text {familiar }}\right) /\left(T_{\text {novel }}+T_{\text {familiar }}\right)$, where $T_{\text {novel }}$ and $T_{\text {familiar }}$ indicate the exploration time during testing for the novel and familiar objects, respectively.

\section{Morris water maze test}

The MWM, a widely accepted method for analyzing spatial learning and memory abilities ${ }^{74}$, was performed. Briefly, the apparatus consisted of a white plastic pool $(120 \mathrm{~cm}$ in diameter and $50 \mathrm{~cm}$ in depth) filled with water $\left(22 \pm 1{ }^{\circ} \mathrm{C}\right)$, and a transparent escape platform $(10 \mathrm{~cm}$, square) located $0.5 \mathrm{~cm}$ to $1 \mathrm{~cm}$ below the water surface. The white edible pigment was added to the water to make the platform invisible during tests. The pool was divided into four quadrants. Mice were introduced to different quadrants randomly to learn how to find the hidden platform within $60 \mathrm{~s}$ by four extra maze cues placed asymmetrically as spatial references. If the mice failed to find the platform within $60 \mathrm{~s}$, they were placed onto the platform and allowed to stay on top for $15 \mathrm{~s}$ to acclimatize with the surroundings. During the spatial test, all mice were trained for a period of 5 days with four trials per day. At the end of learning, a probe trial was conducted $24 \mathrm{~h}$ later with a hidden platform removed. After the spatial test, the mice were administered another set of four trials per day for 5 days and a 1-day probe trial to assess reversal learning. The mice were trained to relocate the platform in the opposite quadrant for 5 additional days, following by the reversal probe trial $24 \mathrm{~h}$ later, with the hidden platform removed again. All experiments were recorded by a computerized tracking system, which calculated distances moved and latencies required for reaching the platform.

\section{Tissue preparation}

After the behavior test, mice were transcardially perfused with cold phosphate-buffered saline (PBS) under deep anesthesia. Brains were removed and separated into the two hemispheres, which were either fixed in $4 \%$ paraformaldehyde (PFA) or fresh-frozen on ice. The cortex and HPC were dissected out from fresh hemispheres and homogenized in RIPA $(50 \mathrm{mM}$ Tris $\mathrm{HCl}, \mathrm{pH}=8.0$, $150 \mathrm{mM} \mathrm{NaCl}, 1 \% \mathrm{NP}-40,0.5 \%$ sodium deoxycholate, $0.1 \%$ SDS) buffer which contained protease and phosphatase inhibitor. The homogenates were incubated on ice for $20 \mathrm{~min}$ and centrifuged at $18,000 \times g$ for $20 \mathrm{~min}$ before supernatants were collected and stored at $-80^{\circ} \mathrm{C}$. Fixed hemispheres were kept in $4 \%$ PFA at $4{ }^{\circ} \mathrm{C}$ for $24 \mathrm{~h}$ and transferred to $30 \%$ sucrose solutions in PBS for $48 \mathrm{~h}$. They were then subsequently snap-frozen in isopentane and stored at $-80^{\circ} \mathrm{C}$ until processing. 


\section{Immunofluorescence}

The brains were coronally sectioned on a freezing microtome at $20 \mu \mathrm{m}$ thickness. For immunofluorescence, sections were blocked in $5 \%$ bovine serum albumin with $0.3 \%$ Triton X-100 for $60 \mathrm{~min}$ followed by overnight incubation with the primary antibody at $4{ }^{\circ} \mathrm{C}$. The primary antibodies were anti-A $\beta \quad(1: 100,8243$, Cell Signaling Technology), anti-A $\beta$ antibody $(1: 400,803004$, Biolegend), anti-Iba1 antibody (1:1000, 019-1947, Wako), antiGFAP antibody (1:1000, MAB360, EMD Millipore), antiGlut1 antibody (1:100, ab40084, Abcam), anti-COX-2 antibody (1:200, 12282, Cell Signaling Technology), and anti-CD163 antibody (1:200, ab182422, Abcam). Thereafter, sections were incubated with goat anti-rabbit IgG Alexa Fluor 488 antibody (1:500, ab150077, Abcam), donkey anti-rabbit IgG Alexa Fluor 555 antibody (1:500, ab150074, Abcam), donkey anti-mouse IgG Alexa Fluor 488 antibody (1:500, ab150105, Abcam) or Goat antimouse IgG HRP-conjugated antibody (1:500, 405306, Biolegend) for $1 \mathrm{~h}$ at room temperature. The sections were mounted through a mounting medium with DAPI (H-1200, Vector laboratories). Images were acquired by a confocal microscope (SP8, Leica) with a 40X objective at identical settings for all conditions.

\section{Histological analysis}

Images were analyzed using Image software (National Institutes of Health, USA). For CA1 imaging, one field of view $(293 \times 293 \mu \mathrm{m})$ per mouse was measured. The cortex was analyzed by measuring the alteration in random 4-6 fields of view $(293 \times 293 \mu \mathrm{m})$ in the cortex per mouse. The morphology of microglia was measured by ImageJ. Meanwhile, the Coloc2 plugin was used to quantify the colocalization of $A \beta$ with microglia and astrocytes. Threedimensional reconstruction and volume of microglia were analyzed using IMARIS software (Bitplane). The COX-2 positive microglia per field of view were counted by an experimenter blind to treatment groups. For vesselassociated microglia, three randomly selected vessels per field of view in the cortex were analyzed. The perivascular microglia were quantified using the Leica Microscope Imaging Software by counting and comparing cells associated and not associated with blood vessels.

\section{Western blot}

For Western blotting, the total protein of each sample was measured by the BCA protein assay kit (Thermo Scientific, Rockford, USA). The protein from each sample was separated by $4-12 \%$ NuPAGE (180-8018H, Tanon). After transfer of proteins to polyvinylidene fluoride membrane (Millipore, Billerica, USA), the membrane was blocked with $5 \%$ skim milk for $1.5 \mathrm{~h}$ at room temperature and incubated for $2 \mathrm{~h}$ at room temperature with the primary antibodies: anti-VEGF antibody (1:1000, 19003-1-
AP, Proteintech), anti-PI3K antibody (1:1000, 4257, Cell Signaling Technology), anti-p-PI3K antibody (1:1000, 4228, Cell Signaling Technology), anti-AKT antibody (1:1000, 4691, Cell Signaling Technology), anti-p-AKT antibody (1:2000, $4060 \mathrm{~T}$, Cell Signaling Technology), anti-ERK antibody (1:1000, 4695, Cell Signaling Technology), anti-p-ERK antibody (1:2000, 4370, Cell Signaling Technology), and anti-GAPDH antibody (1:10000, 600041-Ig, Proteintech). Thereafter, the membrane was incubated with HRP-conjugated secondary antibody at room temperature for $1 \mathrm{~h}$ and treated with enhanced chemiluminescent reagent kit (Thermo Scientific, Rockford, USA). The bands were scanned and digitalized. The density of each band was quantified using Image and normalized to the values of GAPDH.

\section{Statistical analysis}

Data were shown as mean \pm standard error of the mean. The statistical analysis of the data was analyzed using GraphPad Prism 8.0. Comparisons for normally distributed data with two groups were analyzed by two-tailed unpaired t-tests. Comparisons for normally distributed data with three or more groups were tested by one-way ANOVA. Mann-Whitney tests were applied to determine statistical significances of data with the non-normally distribution. Statistical differences for all tests were considered significant at $p<0.05$.

\section{Acknowledgements}

This work was supported by grants from the National Key Research and Development Program of China (Grant No. 2019YFC1604604), the Special Fund for Research on National Major Research Instruments of China (Grant No. 62027824), the National Science Fund for Distinguished Young Scholars (Grant No. 61425006), the SJTU Medicine Engineering Interdisciplinary Research Fund (Grant No. YG2017MS19), the Program of Shanghai Technology Research Leader (Grant No. 17XD1402200), the Fundamental Research Funds for the Central Universities (Grant No. 81661168014), the project of Innovative

Research Team of High-level Local Universities in Shanghai, Talents Program of Shanghai Municipal Health Commission (Chen Liang, Grant No. 2018BR06) and the National Natural Science Foundation of China (Grant No. 92068111, 81973272, 82073836, 61975118, and 62075013). We thank Professor Hao He and Professor Bobo Gu for helpful discussions. We thank Meilu Tian for improving the grammar of the manuscript. We also thank Professor Guoyuan Yang and his students for providing the MWM.

\footnotetext{
Author details

'State Key Laboratory of Oncogenes and Related Genes, Shanghai Cancer Institute, Med-X Research Institute and School of Biomedical Engineering, Shanghai Jiao Tong University, Shanghai 200030, China. Institute of Medical Technology, Peking University Health Science Center, Beijing 100191, China. ${ }^{3}$ Department of Radiology, Peking University Third Hospital, Beijing 100191, China. ${ }^{4}$ Key Lab of Magnetic Resonance Imaging Device and Technique, Beijing 100191, China. ${ }^{5}$ Zhejiang Brainhealth Medical Technology Co., Ltd, Hangzhou 314400, China. ${ }^{6}$ Biomedical Pioneering Innovation Center, Peking University, Beijing 100871, China. ${ }^{7}$ School of Life Sciences, Peking University, Beijing 100871, China. ${ }^{8}$ Department of Neurosurgery, Huashan Hospital, Shanghai Medical College, Fudan University, Shanghai 200040, China. 'Tianqiao and Chrissy Chen Institute for Clinical Translational Research, Huashan Hospital, Shanghai 200040, China. ${ }^{10}$ Department of Pharmacology and Chemical Biology, State Key Laboratory of Oncogenes and Related Genes, Shanghai Universities Collaborative Innovation Center for Translational Medicine, Shanghai Jiao Tong University School of Medicine, Shanghai 200025,
} 
China. ${ }^{11}$ Biomedical Engineering Department, Peking University, Beijing 100081, China. ${ }^{12}$ Key Laboratory of Carcinogenesis and Translational Research (Ministry of Education/Beijing), Peking University Cancer Hospital \& Institute, Beijing 100142, China

\section{Author contributions}

L.T., X.W., and X.G. conceived and designed the research. L.T., Q.L., and F.Z. performed the experiments. L.T., Y.F., X.Z., and X.W. processed images and analyzed the experimental data and results. L.T. wrote the manuscript. H.H., Y.H., and Y.S. revised the manuscript. X.W., X.G., and L.C. supervised the project.

\section{Conflict of interest}

The authors declare no competing interests.

Supplementary information The online version contains supplementary material available at https://doi.org/10.1038/s41377-021-00617-3.

Received: 13 April 2021 Revised: 29 July 2021 Accepted: 17 August 2021 Published online: 08 September 2021

\section{References}

1. Disner, S. G., Beevers, C. G. \& Gonzalez-Lima, F. Transcranial laser stimulation as neuroenhancement for attention bias modification in adults with elevated depression symptoms. Brain Stimul. 9, 780-787 (2016).

2. Huisa, B. N. et al. Transcranial laser therapy for acute ischemic stroke: a pooled analysis of NEST-1 and NEST-2. Int. J. Stroke 8, 315-320 (2013).

3. Meng, C. B., He, Z. Y. \& Xing, D. Low-level laser therapy rescues dendrite atrophy via upregulating BDNF expression: implications for Alzheimer's disease. J. Neurosci. 33, 13505-13517 (2013).

4. Tatmatsu-Rocha, J. C. et al. Mitochondrial dynamics (fission and fusion) and collagen production in a rat model of diabetic wound healing treated by photobiomodulation: comparison of $904 \mathrm{~nm}$ laser and $850 \mathrm{~nm}$ light-emitting diode (LED). J. Photochem. Photobiol. B: Biol. 187, 41-47 (2018).

5. Peoples, C. et al. Photobiomodulation enhances nigral dopaminergic cell survival in a chronic MPTP mouse model of Parkinson's disease. Park. Relat. Disord. 18, 469-476 (2012).

6. Chung, $\mathrm{H}$. et al. The nuts and bolts of low-level laser (light) therapy. Ann. Biomed. Eng. 40, 516-533 (2012).

7. Hamblin, M. R. Photobiomodulation for traumatic brain injury and stroke. J. Neurosci. Res. 96, 731-743 (2018).

8. Karu, T. Primary and secondary mechanisms of action of visible to near-IR radiation on cells. J. Photochem. Photobiol. B: Biol. 49, 1-17 (1999).

9. Karu, T. I. \& Kolyakov, S. F. Exact action spectra for cellular responses relevant to phototherapy. Photomed. Laser Surg. 23, 355-361 (2005).

10. Karu, T. I., Pyatibrat, L. V. \& Afanasyeva, N. I. A novel mitochondrial signaling pathway activated by visible-to-near infrared radiation. Photochem. Photobiol. 80, 366-372 (2004).

11. Chen, A. C. et al. Low-level laser therapy activates NF-kB via generation of reactive oxygen species in mouse embryonic fibroblasts. PLOS ONE 6, e22453 (2011).

12. Liang, J. G., Liu, L. \& Xing, D. Photobiomodulation by low-power laser irradiation attenuates A -induced cell apoptosis through the Akt/GSK3 $\beta /$ B-catenin pathway. Free Radic. Biol. Med. 53, 1459-1467 (2012).

13. Zhang, J. T., Xing, D. \& Gao, X. J. Low-power laser irradiation activates Src tyrosine kinase through reactive oxygen species-mediated signaling pathway. J. Cell. Physiol. 217, 518-528 (2008).

14. Purushothuman, S. et al. Photobiomodulation with near infrared light mitigates Alzheimer's disease-related pathology in cerebral cortex - evidence from two transgenic mouse models. Alzheimer's Res. Ther. 6, 2 (2014).

15. Grillo, S. L. et al. Non-invasive infra-red therapy $(1072 \mathrm{~nm})$ reduces $\beta$-amyloid protein levels in the brain of an Alzheimer's disease mouse model, TASTPM. J. Photochem. Photobiol. B: Biol. 123, 13-22 (2013).

16. Farfara, D. et al. Low-level laser therapy ameliorates disease progression in a mouse model of Alzheimer's disease. J. Mol. Neurosci. 55, 430-436 (2015).

17. De Taboada, L. et al. Transcranial laser therapy attenuates amyloid- $\beta$ peptide neuropathology in amyloid- $\beta$ protein precursor transgenic mice. J. Alzheimer's Dis. 23, 521-535 (2011).
18. Comerota, M. M., Krishnan, B. \& Taglialatela, G. Near infrared light decreases synaptic vulnerability to amyloid beta oligomers. Sci. Rep. 7, 15012 (2017).

19. Purushothuman, S. et al. Near infrared light mitigates cerebellar pathology in transgenic mouse models of dementia. Neurosci. Lett. 591, 155-159 (2015).

20. Berk, C., Paul, G. \& Sabbagh, M. Investigational drugs in Alzheimer's disease: current progress. Expert Opin. Investig. Drugs 23, 837-846 (2014).

21. Vermunt, L. et al. Duration of preclinical, prodromal, and dementia stages of Alzheimer's disease in relation to age, sex, and APOE genotype. Alzheimer's Dement. 15, 888-898 (2019).

22. Hardy, J. A. \& Higgins, G. A. Alzheimer's disease: the amyloid cascade hypothesis. Science 256, 184-185 (1992).

23. Saltmarche, A. E. et al. Significant improvement in cognition in mild to moderately severe dementia cases treated with transcranial plus intranasal photobiomodulation: case series report. Photomed. Laser Surg. 35, 432-441 (2017).

24. Berman, M. H. et al. Photobiomodulation with near infrared light helmet in a pilot, placebo controlled clinical trial in dementia patients testing memory and cognition. J. Neurol. Neurosci. 8, 176 (2017).

25. Han, M. M. et al. Near infra-red light treatment of Alzheimer's diseaseJ. Innov. Opt. Health Sci. 11, 1750012 (2018).

26. Vargas, E. et al. Beneficial neurocognitive effects of transcranial laser in older adults. Lasers Med. Sci. 32, 1153-1162 (2017).

27. laccarino, H. F. et al. Gamma frequency entrainment attenuates amyloid load and modifies microglia. Nature 540, 230-235 (2016).

28. Ando, T. et al. Comparison of therapeutic effects between pulsed and continuous wave 810-nm wavelength laser irradiation for traumatic brain injury in mice. PLOS ONE 6, e26212 (2011).

29. Salehpour, F. et al. Transcranial near-infrared photobiomodulation attenuates memory impairment and hippocampal oxidative stress in sleep-deprived mice. Brain Res. 1682, 36-43 (2018).

30. Dong, T. T. et al. Low-level light in combination with metabolic modulators for effective therapy of injured brain. J. Cereb. Blood Flow. Metab. 35, 1435-1444 (2015).

31. Sasaguri, H. et al. APP mouse models for Alzheimer's disease preclinical studies. EMBO J. 36, 2473-2487 (2017).

32. Bolmont, $T$. et al. Dynamics of the microglial/amyloid Interaction indicate a role in plaque maintenance. J. Neurosci. 28, 4283-4292 (2008).

33. D'Andrea, M. R., Cole, G. M. \& Ard, M. D. The microglial phagocytic role with specific plaque types in the Alzheimer disease brain. Neurobiol. Aging 25, 675-683 (2004).

34. Hansen, D. V., Hanson, J. E. \& Sheng, M. Microglia in Alzheimer's disease. J. Cell Biol. 217, 459-472 (2018).

35. Ries, M. \& Sastre, M. Mechanisms of $A \beta$ clearance and degradation by glial cells. Front. Aging Neurosci. 8, 160 (2016).

36. Bamberger, M. E. et al. A cell surface receptor complex for fibrillar $\beta$-amyloid mediates microglial activation. J. Neurosci. 23, 2665-2674 (2003).

37. Webster, S. D. et al. Complement component C1q modulates the phagocytosis of A $\beta$ by microglia. Exp. Neurol. 161, 127-138 (2000).

38. Jones, R. S. et al. Amyloid- $\beta$-induced astrocytic phagocytosis is mediated by CD36, CD47 and RAGE. J. Neuroimmune Pharmacol. 8, 301-311 (2013).

39. Lee, S. J., Seo, B. R. \& Koh, J. Y. Metallothionein-3 modulates the amyloid $\beta$ endocytosis of astrocytes through its effects on actin polymerization. Mol. Brain 8, 84 (2015).

40. Wegiel, J. et al. The role of microglial cells and astrocytes in fibrillar plaque evolution in transgenic APP 5 mice. Neurobiol. Aging 22, 49-61 (2001).

41. Sastre, M., Klockgether, T. \& Heneka, M. T. Contribution of inflammatory processes to Alzheimer's disease: molecular mechanisms. Int. J. Dev. Neurosci. 24, 167-176 (2006).

42. Squire, L. R. et al. Memory consolidation. Cold Spring Harb. Perspect. Biol. 7, a021766 (2015).

43. Dubois, B. et al. Preclinical Alzheimer's disease: definition, natural history, and diagnostic criteria. Alzheimer's Dement. 12, 292-323 (2016).

44. Davalos, D. et al. ATP mediates rapid microglial response to local brain injury in vivo. Nat. Neurosci. 8, 752-758 (2005).

45. Haynes, S. E. et al. The $P_{2} Y_{12}$ receptor regulates microglial activation by extracellular nucleotides. Nat. Neurosci. 9, 1512-1519 (2006).

46. $\mathrm{Hu}, \mathrm{X} . \mathrm{M}$. et al. Microglial and macrophage polarization-new prospects for brain repair. Nat. Rev. Neurol. 11, 56-64 (2015).

47. David, S. \& Kroner, A. Repertoire of microglial and macrophage responses after spinal cord injury. Nat. Rev. Neurosci. 12, 388-399 (2011). 
48. Jimenez, S. et al. Inflammatory response in the hippocampus of PS1 ${ }_{\mathrm{M} 146 \mathrm{~L}}$ / $\mathrm{APP}_{7515 \mathrm{~L}}$ mouse model of Alzheimer's disease: age-dependent switch in the microglial phenotype from alternative to classic. J. Neurosci. 28, 11650-11661 (2008).

49. Cherry, J. D., Olschowka, J. A. \& O'Banion, M. K. Neuroinflammation and M2 microglia: the good, the bad, and the inflamed. J. Neuroinflammation 11, 98 (2014).

50. Ahmed, A. et al. Minocycline modulates microglia polarization in ischemiareperfusion model of retinal degeneration and induces neuroprotection. Sci. Rep. 7, 14065 (2017).

51. Stankovic, N. D. et al. Microglia-blood vessel interactions: a double-edged sword in brain pathologies. Acta Neuropathol. 131, 347-363 (2016).

52. Hohman, T. J., Bell, S. P. \& Jefferson, A. L. The role of vascular endothelial growth factor in neurodegeneration and cognitive decline: exploring interactions with biomarkers of Alzheimer disease. JAMA Neurol. 72, 520-529 (2015).

53. Almalki, S. G. \& Agrawal, D. K. ERK signaling is required for VEGF-ANEGFR2induced differentiation of porcine adipose-derived mesenchymal stem cells into endothelial cells. Stem Cell Res. Ther. 8, 113 (2017).

54. Karar, J. \& Maity, A. PI3K/AKT/mTOR pathway in angiogenesis. Front. Mol. Neurosci. 4, 51 (2011).

55. Martorell, A. J. et al. Multi-sensory gamma stimulation ameliorates Alzheimer'sassociated pathology and improves cognition. Cell 177, 256-271 (2019). e22.

56. Yang, W. Z. et al. Effects of low power laser irradiation on intracellular calcium and histamine release in RBL-2H3 mast cells. Photochem. Photobiol. 83 979-984 (2007).

57. Gu, Q. B. et al. Stimulation of TRPV1 by green laser light. Evid. Based Complementary Altern. Med. 2012, 857123 (2012).

58. Ryu, J. J. et al. Laser modulation of heat and capsaicin receptor TRPV1 leads to thermal antinociception. J. Dent. Res. 89, 1455-1460 (2010).

59. Albert, E. S. et al. TRPV4 channels mediate the infrared laser-evoked response in sensory neurons. J. Neurophysiol. 107, 3227-3234 (2012).

60. Das, $P$. et al. Transient pharmacologic lowering of $A \beta$ production prior to deposition results in sustained reduction of amyloid plaque pathology. Mol. Neurodegeneration 7, 39 (2012).

61. Davies, S. D. et al. Microglia show altered morphology and reduced arborization in human brain during aging and Alzheimer's disease. Brain Pathol. 27, 795-808 (2017).
62. Qiu, W. Q. et al. Insulin-degrading enzyme regulates extracellular levels of amyloid $\beta$-protein by degradation. J. Biol. Chem. 273, 32730-32738 (1998).

63. Frautschy, S. A. et al. Microglial response to amyloid plaques in APPsw transgenic mice. Am. J. Pathol. 152, 307-317 (1998).

64. Pomilio, C. et al. Glial alterations from early to late stages in a model of Alzheimer's disease: evidence of autophagy involvement in $A \beta$ internalization. Hippocampus 26, 194-210 (2016).

65. Hellwig, S. et al. Forebrain microglia from wild-type but not adult 5xFAD mice prevent amyloid- $\beta$ plaque formation in organotypic hippocampal slice cultures. Sci. Rep. 5, 14624 (2015).

66. Hickman, S. E., Allison, E. K. \& El Khoury, J. Microglial dysfunction and defective $\beta$-amyloid clearance pathways in aging Alzheimer's disease mice. J. Neurosci. 28, 8354-8360 (2008).

67. Ketz, A. K. et al. Characterization of Macrophage/Microglial activation and effect of photobiomodulation in the spared nerve injury model of neuropathic pain. Pain. Med. 18, 932-946 (2017).

68. von Leden, R. E. et al. $808 \mathrm{~nm}$ wavelength light induces a dose-dependent alteration in microglial polarization and resultant microglial induced neurite growth. Lasers Surg. Med. 45, 253-263 (2013).

69. Hickman, S. E. \& El Khoury, J. Mechanisms of mononuclear phagocyte recruitment in Alzheimer's disease. CNS Neurol. Disord. Drug Targerts 9, 168-173 (2010).

70. Farfara, D., Lifshitz, V. \& Frenkel, D. Neuroprotective and neurotoxic properties of glial cells in the pathogenesis of Alzheimer's disease. J. Cell. Mol. Med. 12, 762-780 (2008).

71. das Neves, L. M. S. et al. Laser photobiomodulation (830 and $660 \mathrm{~nm}$ ) in mast cells, VEGF, FGF, and CD34 of the musculocutaneous flap in rats submitted to nicotine. Lasers Med. Sci. 32, 335-341 (2017).

72. Kawano, Y. et al. The production of VEGF involving MAP kinase activation by low level laser therapy in human granulosa cells. Laser Ther. 21, 269-274 (2012).

73. Leger, M. et al. Object recognition test in mice. Nat. Protoc. 8, 2531-2537 (2013).

74. Vorhees, C. V. \& Williams, M. T. Morris water maze: procedures for assessing spatial and related forms of learning and memory. Nat. Protoc. 1, 848-858 (2006). 